\title{
Periodic Solutions of abstract neutral functional differential equations
}

\author{
Bahloul Rachid ${ }^{1}$,Sidki Omar ${ }^{1}$ \\ ${ }^{1}$ Faculty of sciences and Technology, Fes-Saiss Fes, Morocco
}

\section{Abstract}

We charaterize the existence of periodic solutions for a class of abstract neutral functional differential equations described in

$$
\begin{gathered}
\text { the form : } \\
\frac{d}{d t} x(t)=A[x(t)-B x(t-r)]+L\left(x_{t}\right)+f(t), t \in R
\end{gathered}
$$

Keywords : functional differential equations

\section{Introduction :}

Let $\mathrm{X}$ be a Banach space endowed with a norm |.| and $\mathrm{r}$ be non negative real number.

The main objective of this paper is to study the existence of periodic solutions for the class of linear abstract neutral differential equations (1) :

$\mathrm{C}=\mathrm{C}([-\mathrm{r}, 0] ; \mathrm{X})$ be the Banach space of continuous functions mapping the interval $[-\mathrm{r}, 0]$ into $\mathrm{X}$. the function $x_{t}$ given by $x_{t}(\theta)=\mathrm{x}(\mathrm{t}+\theta)$ for $\theta$ in appropriate domain,denotes the segment or the "history" of the function $\mathrm{x}($. at t.

$\mathrm{L}$ is a bounded linear map defined on an appropriate space, and $\mathrm{f}: \mathrm{R} \rightarrow$ $\mathrm{X}$ is a locally $\mathrm{p}$-integrable and $2 \pi$-periodic function for $1 \leq \mathrm{p} \prec+\infty$

we assume that $\mathrm{A}: \mathrm{D}(\mathrm{A}) \subseteq \mathrm{X} \rightarrow \mathrm{X}$ and $\mathrm{B} \subseteq \mathrm{X} \rightarrow \mathrm{X}$ are closed linear operator

We denote

$$
H^{1, p}(T ; \mathrm{X})=\left\{u \in L^{p}(T: X): \ni v \in L^{p}(T ; X), \hat{v}(k)=i k \hat{u}(k) \text { forallk } \in Z\right\}
$$




\section{Preliminaries :}

We denote by $T$ the group defined as the quotient $\mathrm{R} / 2 \pi \mathrm{Z}$. There is an obvious identification between functions on $T$ and $2 \pi$-periodic functions on R. We consider the interval $[0,2 \pi)$ as a model for $T$.

For a function $\mathrm{f} \in L^{1}(T ; \mathrm{X})$, we denote by $\hat{f}(\mathrm{k}), \mathrm{k} \in \mathrm{Z}$ the $\mathrm{k}$-th Fourier coefficient of $\mathrm{f}$ :

$$
\hat{f}(\mathrm{k})=\frac{1}{2 \pi} \int_{0}^{1} e^{-i k t} \mathrm{f}(\mathrm{t}) \mathrm{dt} \text { for } \mathrm{k} \in \mathrm{Z} \text { and } \mathrm{t} \in \mathrm{R} \text {. }
$$

Denote $f_{\tau}(\mathrm{t}):=\mathrm{f}(\mathrm{t}+\tau), \tau \in \mathrm{Z}$; then it the follows from the definition that $\hat{f}_{\tau}(\mathrm{k})=e^{i k \tau} \hat{f}(\mathrm{k}), \tau \in T$.

Let $\mathrm{f} \in L^{p}(T, \mathrm{X})$. Then by Fefer's theorem, one has

$$
\mathrm{f}=\lim _{n \rightarrow \infty} \sigma_{n}(\mathrm{f})
$$

in $L^{p}(T, \mathrm{X})$ where

$$
\sigma_{n}(\mathrm{f}):=\frac{1}{n+1} \sum_{m=0}^{n} \sum_{k=-m}^{m} e_{k} \hat{f}(\mathrm{k})
$$

with $e_{k}(\mathrm{t}):=e^{i k t}$

A Banach space $\mathrm{X}$ is said to be UMD, if the Hilbert transform is bounded on $L^{p}(R, \mathrm{X})$ for all $\mathrm{p} \in(1, \infty)$.

Definition 1 : Let $X$ and $Y$ be a Banach spaces. A family of operators $T \subset B(X, Y)$ is called $R$-bounded, if there is a constant $C \succ 0$ and $p \in[1, \infty)$ such that for each $N \in N, T_{j} \in T, x_{j} \in X$ and for all independent, symmetric, $\{-1,1\}$ valued random variables $r_{j}$ on a probability space $(\Omega, M, \mu)$ the inequality $\left\|\sum_{j=1}^{N} r_{j} T_{j} x_{j}\right\|_{L^{p}(\Omega, Y)} \leq C\left\|\sum_{j=1}^{N} r_{j} x_{j}\right\|_{L^{p}(\Omega, Y)}$ is valid. The smallest such $C$ is called R-bounded of $T$, we denot it by $R_{p}(T)$. 
Definition 2 : For $1 \leq p \infty$ we say that a sequence $\left\{M_{k}\right\}_{k \in Z} \subset B(X, Y)$ is an $L^{p}$-multiplier if, for each $f \in L^{p}(T, X)$, there exists $u \in L^{p}(T, Y)$ such that $\hat{u}(k)=M_{k} \hat{f}(k)$ for all $k \in Z$.

Theorem $1:[3$, theorem 1.3$]$

Let $X, Y$ be UMD space and let $\left\{M_{k}\right\}_{k \in Z} \subset B(X, Y)$. If the sets $\left\{M_{k}\right\}_{k \in Z}$ and $\left\{k\left(M_{k+1}-M_{k}\right)\right\}_{k \in Z}$ are $R$-bounded, then $\left\{M_{k}\right\}_{k \in Z}$ is an $L^{p}$-multiplier for 1 $\prec p \prec \infty$.

\section{A Criterion for Periodic Solutions :}

We consider : $\Delta_{k}=\mathrm{ikI}-\mathrm{ik} B_{k}-\mathrm{A}\left(\mathrm{I}-B_{k}\right)-L_{k}$, for all $\mathrm{k} \in \mathrm{Z}$.

Denote by $B_{k}:=e^{-i k r} \mathrm{~B} ; L_{k}(\mathrm{x}):=\mathrm{L}\left(e^{i k \theta} \mathrm{x}\right)$ and $e_{k}(\mathrm{t}):=e^{i k t}$ for all $\mathrm{k} \in \mathrm{Z}$ and $\sigma_{Z}(\Delta)=\left\{k \in Z: \Delta_{k}\right.$ has no inverse $\}$

And we define : $D_{k}=\left(i k I-A\left(I-B_{k}\right)-L_{k}\right)^{-1}$

3.1. Existence of Strong Solution :

Definition 3 Let A be a closed linear operator on X. A function x(.) solution of the problem (1) if $x \in H^{1, p}(T ; X) \cap L^{p}(T ; X)$ and (1) holds for almost all $t \in[0,2 \pi]$ 
Theorem 2 : Let $X$ be a Banach space and $1 \prec p \prec+\infty$. Suppose that for every $f \in L^{p}(T, X)$ there exists a unique strong solution of $E q$ (1).Then

1. for every $k \in Z$ the operator ( $i k I-A\left(I-B_{k}\right)-L_{k}$ ) has bounded inverse

2. The set is R-bounded and $\left\{i k D_{k}\right\}_{k \in Z}$ is R-bounded.

Lemma 1 :[2, Lemma 4.2]

Let $u \in C(T, X)$. Then

$$
\hat{L\left(X_{s}(k)\right.}=L_{k} \hat{x}(k) .
$$

proof of theorem 2:

1) Let $k \in Z, y \in X$

$$
\begin{aligned}
& \text { for } \mathrm{f}(\mathrm{t})=e^{i k r} \mathrm{y}, \exists x \in H^{1, p}(T, X) \text { such that : } \\
& \frac{d x}{d t}(\mathrm{t})=\mathrm{A}(\mathrm{x}(\mathrm{t})-\mathrm{Bx}(\mathrm{t}-\mathrm{r}))+\mathrm{L}\left(x_{t}\right)+\mathrm{f}(\mathrm{t})
\end{aligned}
$$

Taking fourier transform, L is linear and bounded, we obtain

$$
\begin{aligned}
& \mathrm{ik} \hat{x}(\mathrm{k})=\mathrm{A}\left(\mathrm{I}-B_{k}\right) \hat{x}(\mathrm{k})+L_{k} \hat{x}(\mathrm{k})+\hat{f}(\mathrm{k}) \\
& \left.\left(\mathrm{ikI}-\mathrm{A}\left(\mathrm{I}-B_{k}\right)-L_{k}\right) \hat{x}(\mathrm{k})=\hat{f}(\mathrm{k})=\mathrm{y} \Rightarrow\right)\left(\mathrm{ikI}-\mathrm{A}\left(\mathrm{I}-B_{k}\right)-L_{k}\right) \text { is surjective. }
\end{aligned}
$$

Let $\mathrm{x} \in \operatorname{Ker}\left(\left(\mathrm{ik}-\mathrm{A}\left(\mathrm{I}-B_{k}\right)-L_{k}\right)\right)$, that is $\mathrm{A}\left(\mathrm{I}-B_{k}\right) \mathrm{x}+L_{k} \mathrm{x}=\mathrm{ikx}$, then $\mathrm{u}(\mathrm{t})=e_{k} \mathrm{x}$ defines a periodic solution of $(1)$ corresponding to the the function $\mathrm{f}(\mathrm{t})=0$.Consequently, $\mathrm{u}(\mathrm{t})=0$ and $\mathrm{x}=0$.

2) let $\mathrm{f} \in L^{p}(\mathrm{~T}, \mathrm{X})$. By hypothesis, there exists a unique $\mathrm{x} \in H^{1, p}(\mathrm{~T}, \mathrm{X})$ such that (1) equation is valid.Taking

Fourier transforms, we deduce that (ikI-A $\left.\left(\mathrm{I}-B_{k}\right)-L_{k}\right) \hat{x}(\mathrm{k})=\hat{f}(\mathrm{k})$ for all

$\mathrm{k} \in \mathrm{Z}$. Hence

$\mathrm{ik} \hat{x}(\mathrm{k})=\mathrm{ik}\left(i k I-A\left(I-B_{k}\right)-L_{k}\right)^{-1} \hat{f}(\mathrm{k})$ for all $\mathrm{k} \in \mathrm{Z}$

On the other hand, since $\mathrm{x} \in H^{1, p}(\mathrm{~T}, \mathrm{X})$, there exists $\mathrm{v} \in L^{p}(\mathrm{~T}, \mathrm{X})$ such that $\hat{v}(\mathrm{k})=\mathrm{i} \mathrm{k} \hat{x}(\mathrm{k})$. This proves claim. 
3.2. Existence of weak solution :

Definition 4 : Let $A$ be a closed linear operator on $X$. A function $x($.$) is$ called a weak solution of the problem (1) if : $\int_{0}^{t}(x(s)-B x(s-r)) d s \in D(A)$ and $x(t)-x(0)=A \int_{0}^{t}(x(s)-B x(s-r)) d s+\int_{0}^{t}\left(L x_{s}+f(s)\right) d s, \quad 0 \leq t \leq 2 \pi$.

Theorem 3 : Let $f \in L^{p}(T, X)$, Assume that $\overline{D(A)}=X$; if $x($.$) is said to be a$ weak solution of $E q(1)$ then $\left(i k I-A\left(I-B_{k}\right)-L_{k}\right) \hat{x}(k)=\hat{f}(k)$ for all $k \in Z$ proof : $\mathrm{x}($.$) is a weak solution of \mathrm{Eq}(1)$ then

$$
\begin{aligned}
& \mathrm{x}(\mathrm{t})-\mathrm{x}(0)=\mathrm{A} \int_{0}^{t} \mathrm{Dx}(\mathrm{s}) \mathrm{ds}+\int_{0}^{t}\left(\mathrm{G} x_{s}+\mathrm{f}(\mathrm{s})\right) \mathrm{ds} \\
& \mathrm{t}=2 \pi \\
& \mathrm{x}(2 \pi)-\mathrm{x}(0)=\mathrm{A} \int_{0}^{2 \pi}(\mathrm{x}(\mathrm{s})-\mathrm{Bx}(\mathrm{s}-\mathrm{r})) \mathrm{ds}+\int_{0}^{2 \pi}\left(\mathrm{L} x_{s}+\mathrm{f}(\mathrm{s})\right) \mathrm{ds} ; \text { or } \mathrm{x}(2 \pi)=\mathrm{x}(0)
\end{aligned}
$$
then

$$
\mathrm{A} \int_{0}^{2 \pi}(\mathrm{x}(\mathrm{s})-\mathrm{Bx}(\mathrm{s}-\mathrm{r})) \mathrm{ds}+\int_{0}^{2 \pi}\left(\mathrm{L} x_{s}+\mathrm{f}(\mathrm{s})\right) \mathrm{ds}=0
$$

$\left(\mathrm{A} I-B_{0}+L_{0}\right) \hat{x}(0)+\hat{f}(0)=0$

$\left(0-\mathrm{A} I-B_{0^{-}} L_{0}\right) \hat{x}(0)=\hat{f}(0)$ which shows that the assertion holds for $\mathrm{k}=0$.

Define $\mathrm{v}(\mathrm{t})=\int_{0}^{t}(\mathrm{x}(\mathrm{s})-\mathrm{Bx}(\mathrm{s}-\mathrm{r})) \mathrm{ds}$

And $g(\mathrm{t})=\mathrm{x}(\mathrm{t})-\mathrm{x}(0)-\int_{0}^{t}\left(\mathrm{~L} x_{s}+\mathrm{f}(\mathrm{s})\right) \mathrm{ds}$

by lemma $3.1[2]$

We have $\hat{v}(\mathrm{k})=\frac{i}{k}(\hat{x}(0)-\mathrm{B} \hat{x}(0))-\frac{i}{k}(\hat{x}(\mathrm{k})-\mathrm{B} \hat{x}(\mathrm{k}))(\operatorname{remark} 2.3[2])$

$$
\begin{aligned}
& \hat{g}(\mathrm{k})=\hat{x}(\mathrm{k})-\left[\frac{i}{k} L_{0} \hat{x}(0)-\frac{i}{k} L_{k} \hat{x}(\mathrm{k})\right]-\left[\frac{i}{k} \hat{f}(0)-\frac{i}{k} \hat{f}(\mathrm{k})\right] \\
& \hat{g}(\mathrm{k})=\hat{x}(\mathrm{k})-\frac{i}{k} L_{0} \hat{x}(0)+\frac{i}{k} L_{k} \hat{x}(\mathrm{k})-\frac{i}{k} \hat{f}(0)+\frac{i}{k} \hat{f}(\mathrm{k}) \\
& \mathrm{A} \hat{v}(\mathrm{k})=\frac{i}{k} \mathrm{~A}\left(\mathrm{I}-B_{0}\right) \hat{x}(0)-\frac{i}{k} \mathrm{~A}\left(\mathrm{I}-B_{k} \hat{x}(\mathrm{k})\right.
\end{aligned}
$$


Then

$$
\begin{aligned}
& \mathrm{ik} \hat{x}(\mathrm{k})+L_{0} \hat{x}(0)-L_{k} \hat{x}(\mathrm{k})+\hat{f}(0)-\hat{f}(\mathrm{k})=-\mathrm{A}\left(\mathrm{I}-B_{0}\right) \hat{x}(0)+\mathrm{A}\left(\mathrm{I}-B_{k}\right) \hat{x}(\mathrm{k}) \\
& \Leftrightarrow\left[\mathrm{ik} \hat{x}(\mathrm{k})-\mathrm{A}\left(\mathrm{I}-B_{k}\right) \hat{x}(\mathrm{k})-L_{k} \hat{x}(\mathrm{k})-\hat{f}(\mathrm{k})\right]-\left[\mathrm{A}\left(\mathrm{I}-B_{0}\right) \hat{x}(0)+L_{0} \hat{x}(0)+\hat{f}(0)\right]=0 \\
& \Leftrightarrow \mathrm{ik} \hat{x}(\mathrm{k})-\mathrm{A}\left(\mathrm{I}-B_{k}\right) \hat{x}(\mathrm{k})-L_{k} \hat{x}(\mathrm{k})-\hat{f}(\mathrm{k})=0 \\
& \Leftrightarrow \mathrm{ik} \hat{x}(\mathrm{k})-\mathrm{A}\left(\mathrm{I}-B_{k}\right) \hat{x}(\mathrm{k})-L_{k} \hat{x}(\mathrm{k})=\hat{f}(\mathrm{k}) .
\end{aligned}
$$

Theorem 4 Let $f \in L^{p}(T, X)$, Assume that $\overline{D(A)}=X$; if $x($.$) is said to be a$ weak solution of $E q(2)$ and $\left(i k D_{k}-A D_{k}-G_{k}\right)$ has a bounded inverse. Then $\left(i k I-A\left(I-B_{k}\right)-L_{k}\right)^{-1}$ is an $L^{p}$-multiplier.

proof; from theorem (1) we have $\hat{x}(\mathrm{k})=\left(i k I-A\left(I-B_{k}\right)-L_{k}\right)^{-1} \hat{f}(\mathrm{k})$, for all $\mathrm{f} \in L^{p}(\mathrm{~T}, \mathrm{X})$

\section{Main result :}

Our main result in this paper, establish that the converse of theorem (2) and the give the definition of Mild solution

\section{Theorem 5 :}

Let $X$ be a UMD space and let $A: D(A) \subset X \rightarrow X$ be a closed linear operator. The following assertions are equivalent for $1 \prec p \prec \infty$.

1. for every $f \in L^{p}(T, X)$ there exists a unique strong solution of $E q(1)$

2. for every $k \in Z$ the operator ( $i k I-A\left(I-B_{k}\right)-L_{k}$ ) has bounded inverse and the set is R-bounded and $\left\{i k D_{k}\right\}_{k \in Z}$ is R-bounded. 
proof :

$1 \Leftarrow 2)$ Let $\mathrm{f} \in L^{p}(\mathrm{~T}, \mathrm{X})$. Define $D_{k}=\left(i k I-A\left(I-B_{k}\right)-L_{k}\right)^{-1}$, the family

$\left\{i k D_{k}\right\}_{k \in Z}$ is an $L^{p}$-multiplier it is equivalent to the family $\left\{D_{k}\right\}_{k \in Z}$

is an $L^{p}$-multiplier that maps $L^{p}(\mathrm{~T}, \mathrm{X})$ into $H^{1, p}(\mathrm{~T}, \mathrm{X})$, [ i.e. there exists

$\mathrm{x} \in H^{1, p}(\mathrm{~T}, \mathrm{x})$ such that

$$
\hat{x}(\mathrm{k})=D_{k} \hat{f}(\mathrm{k})=\left(i k I-A\left(I-B_{k}\right)-L_{k}\right)^{-1} \hat{f}(\mathrm{k})
$$

In particular, $\mathrm{x} \in L^{p}(\mathrm{~T}, \mathrm{X})$ and there exists $\mathrm{v} \in L^{p}(\mathrm{~T}, \mathrm{X})$ such that

$$
\hat{x^{\prime}}(\mathrm{k}):=\hat{v}(\mathrm{k})=\mathrm{ik} \hat{x}(\mathrm{k})
$$

By Fejer's theorem one has in $L^{p}\left(\left[-r_{2 \pi}, 0\right], \mathrm{X}\right)$

$$
x_{t}(\theta)=\mathrm{x}(\mathrm{t}+\theta)=\lim _{n \rightarrow+\infty} \frac{1}{n+1} \sum_{m=0}^{n} \sum_{k=-m}^{m} e^{i k t} e^{i k \theta} \hat{x}(\mathrm{k})
$$

Hence in $L^{p}(\mathrm{~T}, \mathrm{X})$ we obtain

$$
x_{t}=\lim _{n \rightarrow+\infty} \frac{1}{n+1} \sum_{m=0}^{n} \sum_{k=-m}^{m} e^{i k t} e_{k} \hat{x}(\mathrm{k})
$$

Then, since $\mathrm{L}$ is linear and bounded

$$
\begin{aligned}
\mathrm{L} x_{t} & =\lim _{n \rightarrow+\infty} \frac{1}{n+1} \sum_{m=0}^{n} \sum_{k=-m}^{m} e^{i k t} \mathrm{~L}\left(e_{k} \hat{x}(\mathrm{k})\right) \\
& =\lim _{n \rightarrow+\infty} \frac{1}{n+1} \sum_{m=0}^{n} \sum_{k=-m}^{m} e^{i k t} L_{k} \hat{x}(\mathrm{k})
\end{aligned}
$$

By (1.1) and (1.2) we have

$$
\hat{x}^{\prime}(\mathrm{k})=\mathrm{ik} \hat{x}(\mathrm{k})=\mathrm{A}\left(\mathrm{I}-B_{k}\right) \hat{x}(\mathrm{k})+L_{k} \hat{x}(\mathrm{k})+\hat{f}(\mathrm{k}) \text {. for all } \mathrm{k} \in \mathrm{Z} \text {. }
$$

Then using that $\mathrm{A}$ and $\mathrm{B}$ are closed we conclude tat $(\mathrm{x}(\mathrm{t})-\mathrm{Bx}(\mathrm{t}-\mathrm{r})) \in \mathrm{D}(\mathrm{A})$, and from the uniqueness theorem of Fourier coefficients, that equation (2) is valid for $\mathrm{t} \in \mathrm{T}$.[3. lemma 3.1] 
Definition 5 : of Mild solution about convert of weak solution

Introduction :

Assume that A generates a $C_{0}$-semigroup $\mathrm{T}($.$) on \mathrm{X}$; and $\mathrm{x}($.$) is a weak$ solution, then we have

$$
\begin{gathered}
\mathrm{x}(\mathrm{t})-\mathrm{x}(0)=\mathrm{A} \int_{0}^{t}(\mathrm{x}(\mathrm{s})-\mathrm{Bx}(\mathrm{s}-\mathrm{r})) \mathrm{ds}+\int_{0}^{t}\left(\mathrm{G} x_{s}+\mathrm{f}(\mathrm{s})\right) \mathrm{ds} \\
\int_{0}^{t} \mathrm{~T}(\mathrm{t}-\mathrm{s})(\mathrm{x}(\mathrm{s})-\mathrm{x}(0)) \mathrm{ds}= \\
\int_{0}^{t} \mathrm{~T}(\mathrm{t}-\mathrm{s}) \mathrm{A} \int_{0}^{s}(\mathrm{x}(\xi)-\mathrm{Bx}(\xi-\mathrm{r})) \mathrm{d} \xi \mathrm{ds}+\int_{0}^{t} \mathrm{~T}(\mathrm{t}-\mathrm{s}) \int_{0}^{s}\left(\mathrm{~L}\left(x_{\xi}\right)+\mathrm{f}(\xi)\right) \mathrm{d} \xi \mathrm{ds} \\
=\int_{0}^{t}(\mathrm{~T}(\mathrm{t}-\mathrm{s})-\mathrm{I})(\mathrm{x}(\mathrm{s})-\mathrm{Bx}(\mathrm{s}-\mathrm{r})) \mathrm{ds}+\int_{0}^{t} \mathrm{~T}(\mathrm{t}-\mathrm{s}) \int_{0}^{s}\left(\mathrm{~L}\left(x_{\xi}\right)+\mathrm{f}(\xi)\right) \mathrm{d} \xi \mathrm{ds}
\end{gathered}
$$

Then

$$
\begin{aligned}
& \int_{0}^{t} \mathrm{~T}(\mathrm{t}-\mathrm{s})(\mathrm{Bx}(\mathrm{s}-\mathrm{r})-\mathrm{x}(0)) \mathrm{ds}=-\int_{0}^{s}(\mathrm{x}(\mathrm{s})-\mathrm{Bx}(\mathrm{s}-\mathrm{r})) \mathrm{ds}+\int_{0}^{t} \mathrm{~T}(\mathrm{t}-\mathrm{s}) \int_{0}^{s}\left(\mathrm{~L}\left(x_{\xi}\right)+\mathrm{f}(\xi)\right) \mathrm{d} \xi \mathrm{ds} \\
& \int_{0}^{t}(\mathrm{x}(\mathrm{s})-\mathrm{Bx}(\mathrm{s}-\mathrm{r})) \mathrm{ds}+\int_{0}^{s} \mathrm{~T}(\mathrm{t}-\mathrm{s})(\mathrm{Bx}(\mathrm{s}-\mathrm{r})-\mathrm{x}(0)) \mathrm{ds}=\int_{0}^{t} \mathrm{~T}(\mathrm{t}-\mathrm{s}) \int_{0}^{s}\left(\mathrm{~L}\left(x_{\xi}\right)+\mathrm{f}(\xi)\right) \mathrm{d} \xi \mathrm{ds}
\end{aligned}
$$

$\mathrm{A} \int_{0}^{t}(\mathrm{x}(\mathrm{s})-\mathrm{Bx}(\mathrm{s}-\mathrm{r})) \mathrm{ds}+\mathrm{A} \int_{0}^{s} \mathrm{~T}(\mathrm{t}-\mathrm{s})(\mathrm{Bx}(\mathrm{s}-\mathrm{r})-\mathrm{x}(0)) \mathrm{ds}=\mathrm{A} \int_{0}^{t} \mathrm{~T}(\mathrm{t}-\mathrm{s}) \int_{0}^{s}\left(\mathrm{~L}\left(x_{\xi}\right)+\mathrm{f}(\xi)\right) \mathrm{d} \xi \mathrm{ds}$

$\mathrm{A} \int_{0}^{t}(\mathrm{x}(\mathrm{s})-\mathrm{Bx}(\mathrm{s}-\mathrm{r})) \mathrm{ds}+\mathrm{A} \int_{0}^{s} \mathrm{~T}(\mathrm{t}-\mathrm{s})(\mathrm{Bx}(\mathrm{s}-\mathrm{r})-\mathrm{x}(0)) \mathrm{ds}=\int_{0}^{t}(\mathrm{~T}(\mathrm{t}-\mathrm{s})-\mathrm{I}) \int_{0}^{s}\left(\mathrm{~L}\left(x_{\xi}\right)+\mathrm{f}(\xi)\right) \mathrm{d} \xi \mathrm{ds}$

$\mathrm{A} \int_{0}^{t}(\mathrm{x}(\mathrm{s})-\mathrm{Bx}(\mathrm{s}-\mathrm{r})) \mathrm{d} \mathrm{s}+\int_{0}^{t}\left(\mathrm{~L}\left(x_{s}\right)+\mathrm{f}(\mathrm{s})\right) \mathrm{ds}=$

$\int_{0}^{t} \mathrm{~T}(\mathrm{t}-\mathrm{s})\left(\mathrm{L}\left(x_{s}\right)+\mathrm{f}(\mathrm{s})\right) \mathrm{ds}+\mathrm{A} \int_{0}^{t} \mathrm{~T}(\mathrm{t}-\mathrm{s})(\mathrm{x}(0)-\mathrm{Bx}(\mathrm{s}-\mathrm{r})) \mathrm{ds}$

or $\mathrm{x}($.$) is a weak solution then$

$$
\mathrm{x}(\mathrm{t})-\mathrm{x}(0)=\mathrm{A} \int_{0}^{t} \mathrm{~T}(\mathrm{t}-\mathrm{s})(\mathrm{x}(0)-\mathrm{Bx}(\mathrm{s}-\mathrm{r})) \mathrm{ds}+\int_{0}^{t} \mathrm{~T}(\mathrm{t}-\mathrm{s})\left(\mathrm{L}\left(x_{s}\right)+\mathrm{f}(\mathrm{s})\right) \mathrm{ds}
$$

Our object, establish the convese of this result

Definition 6 : Assume that $A$ generates a $C_{0}$-semigroup $T($.$) on X$. A function $x($.$) is called a mild solution of the problem (1) if :$

$\int_{0}^{t} T(t-s)(x(0)-B x(s-r)) d s \in D(A)$ and $x(t)-x(0)=A \int_{0}^{t} T(t-s)(x(0)-B x(s-r)) d s+\int_{0}^{t} T(t-s)\left(L\left(x_{s}\right)+f(s)\right) d s \quad 0 \leq t \leq 2 \pi$. 
Corollary 1 Assume that $A$ generates a $C_{0}$-semigroup $T($.$) on X$; let $f \in$ $L^{p}(T, X)$

$x($.$) is a weak solution \Leftrightarrow x($.$) is a mild solution$

proof :

$\Rightarrow$ ) by introduction

$\Leftarrow)$ suppose that $\mathrm{x}($.$) is a mild solution of \mathrm{Eq}(2)$ then

$\mathrm{x}(\mathrm{t})-\mathrm{x}(0)=\mathrm{A} \int_{0}^{t} \mathrm{~T}(\mathrm{t}-\mathrm{s})(\mathrm{x}(0)-\mathrm{Bx}(\mathrm{s}-\mathrm{r})) \mathrm{ds}+\int_{0}^{t} \mathrm{~T}(\mathrm{t}-\mathrm{s})\left(\mathrm{L}\left(x_{s}\right)+\mathrm{f}(\mathrm{s})\right) \mathrm{ds}$

$\int_{0}^{t}(\mathrm{x}(\mathrm{s})-\mathrm{x}(0)) \mathrm{ds}=\int_{0}^{t} \mathrm{~A} \int_{0}^{s} \mathrm{~T}(\mathrm{t}-\xi)(\mathrm{x}(0)-\mathrm{Bx}(\xi-\mathrm{r})) \mathrm{d} \xi \mathrm{ds}+\int_{0}^{t} \int_{0}^{s} \mathrm{~T}(\mathrm{t}-\xi)\left(\mathrm{L}\left(x_{\xi}\right)+\mathrm{f}(\xi)\right) \mathrm{d} \xi \mathrm{ds}$

$\int_{0}^{t}(\mathrm{x}(\mathrm{s})-\mathrm{x}(0)) \mathrm{ds}=\int_{0}^{t}(\mathrm{~T}(\mathrm{t}-\mathrm{s})-\mathrm{I})(\mathrm{x}(0)-\mathrm{Bx}(\mathrm{s}-\mathrm{r})) \mathrm{ds}+\int_{0}^{t} \int_{0}^{s} \mathrm{~T}(\mathrm{t}-\xi)\left(\mathrm{L}\left(x_{\xi}\right)+\mathrm{f}(\xi)\right) \mathrm{d} \xi \mathrm{ds}$

$\mathrm{A} \int_{0}^{t}(\mathrm{x}(\mathrm{s})-\mathrm{x}(0)) \mathrm{ds}=\mathrm{A} \int_{0}^{t}(\mathrm{~T}(\mathrm{t}-\mathrm{s})-\mathrm{I})(\mathrm{x}(0)-\mathrm{Bx}(\mathrm{s}-\mathrm{r})) \mathrm{ds}+\mathrm{A} \int_{0}^{t} \int_{0}^{s} \mathrm{~T}(\mathrm{t}-\xi)\left(\mathrm{L}\left(x_{\xi}\right)+\mathrm{f}(\xi)\right) \mathrm{d} \xi \mathrm{ds}$

$\mathrm{A} \int_{0}^{t}(\mathrm{x}(\mathrm{s})-\mathrm{x}(0)) \mathrm{ds}=\mathrm{A} \int_{0}^{t}(\mathrm{~T}(\mathrm{t}-\mathrm{s})-\mathrm{I})(\mathrm{x}(0)-\mathrm{Bx}(\mathrm{s}-\mathrm{r})) \mathrm{ds}+\int_{0}^{t}(\mathrm{~T}(\mathrm{t}-\mathrm{s})-\mathrm{I})\left(\mathrm{L}\left(x_{s}\right)+\mathrm{f}(\mathrm{s})\right) \mathrm{ds}$

$\mathrm{A} \int_{0}^{t}(\mathrm{x}(\mathrm{s})-\mathrm{x}(0)) \mathrm{ds}+\int_{0}^{t}\left(\mathrm{~L}\left(x_{s}\right)+\mathrm{f}(\mathrm{s})\right) \mathrm{ds}+\mathrm{A} \int_{0}^{t}(\mathrm{x}(0)-\mathrm{Bx}(\mathrm{s}-\mathrm{r})) \mathrm{ds}=\mathrm{A} \int_{0}^{t} \mathrm{~T}(\mathrm{t}-\mathrm{s})(\mathrm{x}(0)-$ $\mathrm{Bx}(\mathrm{s}-\mathrm{r})) \mathrm{ds}+\int_{0}^{t}\left(\mathrm{~T}(\mathrm{t}-\mathrm{s})\left(\mathrm{L}\left(x_{s}\right)+\mathrm{f}(\mathrm{s})\right) \mathrm{ds}\right.$

$$
\underbrace{A \int_{0}^{t} T(t-s)(x(0)-B x(s-r)) d s+\int_{0}^{t}\left(T(t-s)\left(L\left(x_{s}\right)+f(s)\right) d s\right.}_{=x(t)-x(0)}=\mathrm{A} \int_{0}^{t}(\mathrm{x}(\mathrm{s})-
$$

$\mathrm{Bx}(\mathrm{s}-\mathrm{r})) \mathrm{ds}+\int_{0}^{t}\left(\mathrm{~L}\left(x_{s}\right)+\mathrm{f}(\mathrm{s})\right) \mathrm{ds}$

$\mathrm{x}(\mathrm{t})-\mathrm{x}(0)=\mathrm{A} \int_{0}^{t}(\mathrm{x}(\mathrm{s})-\mathrm{Bx}(\mathrm{s}-\mathrm{r})) \mathrm{ds}+\int_{0}^{t}\left(\mathrm{~L}\left(x_{s}\right)+\mathrm{f}(\mathrm{s})\right) \mathrm{ds}$ then $\mathrm{x}($.$) is a weak$ solution.

Proposition 1 : Assume that $A$ generates a $C_{0}$-semigroup $T($.$) on X$. if $\left(i k I-A\left(I-B_{k}\right)-L_{k}\right)^{-1}$ is an $L^{p}$-multiplier Then there exists a unique weak(mild) solution of Eq (1). 
proof : let $\mathrm{f} \in L^{p}(\mathrm{~T}, \mathrm{X})$, then $\mathrm{f}(\mathrm{t})=\lim _{n \rightarrow+\infty} \frac{1}{n+1} \sum_{m=0}^{n} \sum_{k=-m}^{m} e^{i k t} \hat{f}(\mathrm{k})$ or $\left(i k I-A\left(I-B_{k}\right)-L_{k}\right)^{-1}$ is an $L^{p}$-multiplier then there exists $\mathrm{x} \in L^{p}(\mathrm{~T}, \mathrm{X})$ such that $\hat{x}(\mathrm{k})=\left(i k I-A\left(I-B_{k}\right)-L_{k}\right)^{-1} \hat{f}(\mathrm{k})$ put $x_{n}(\mathrm{t})=\lim _{n \rightarrow+\infty} \frac{1}{n+1} \sum_{m=0}^{n} \sum_{k=-m}^{m} e^{i k t}\left(i k I-A\left(I-B_{k}\right)-L_{k}\right)^{-1} \hat{f}(\mathrm{k})$ then $x_{n}(\mathrm{t}) \rightarrow \mathrm{x}(\mathrm{t})$ and $x_{n}$ is strong $L^{p}$-solution of Eq (1) and $x_{n}$ verified

$$
\begin{aligned}
& x_{n}(\mathrm{t})-x_{n}(0)=\mathrm{A} \int_{0}^{t}\left(\left(x_{n}(\mathrm{t}-\mathrm{s})\right)-\mathrm{B} x_{n}(\mathrm{t}-\mathrm{s})\right) \mathrm{ds}+\int_{0}^{t}\left(\mathrm{G}\left(\left(x_{n}\right)_{s}\right)+f_{n}(\mathrm{~s})\right) \mathrm{ds} \\
& \text { we put } y_{n}=x_{n}(0) \text { then } \\
& x_{n}(\mathrm{t})=y_{n}+\mathrm{A} \int_{0}^{t}\left(\left(x_{n}(\mathrm{t}-\mathrm{s})\right)-\mathrm{B} x_{n}(\mathrm{t}-\mathrm{s})\right) \mathrm{ds}+\int_{0}^{t}\left(\mathrm{~L}\left(\left(x_{n}\right)_{s}\right)+f_{n}(\mathrm{~s})\right) \mathrm{ds} \\
& \mathrm{t}=2 \pi \\
& \underbrace{x_{n}(2 \pi)}_{=x_{n}(0)}=y_{n}+\mathrm{A} \int_{0}^{2 \pi}\left(\left(x_{n}(\mathrm{t}-\mathrm{s})\right)-\mathrm{B} x_{n}(2 \pi-\mathrm{s})\right) \mathrm{ds}+\int_{0}^{2 \pi}\left(\mathrm{L}\left(\left(x_{n}\right)_{s}\right)+f_{n}(\mathrm{~s})\right) \mathrm{ds} \\
& (\mathrm{n} \rightarrow \infty) \\
& \quad * \mathrm{y}=\mathrm{y}+\mathrm{A} \int_{0}^{2 \pi}\left((x(s)-\mathrm{Bx}(2 \pi-\mathrm{r})) \mathrm{ds}+\int_{0}^{2 \pi}\left(\mathrm{L}\left(x_{s}\right)+\mathrm{f}(\mathrm{s})\right) \mathrm{ds}\right. \\
& \quad \mathrm{x}(\mathrm{t})=\mathrm{y}+\mathrm{A} \int_{0}^{t}(\mathrm{x}(\mathrm{s})-\mathrm{Bx}(\mathrm{t}-\mathrm{s})) \mathrm{ds}+\int_{0}^{t}\left(\mathrm{~L}\left(x_{s}\right)+\mathrm{f}(\mathrm{s})\right) \mathrm{ds}:=\mathrm{g}(\mathrm{t}) \\
& \quad \mathrm{x}(2 \pi)=\mathrm{g}(2 \pi)=\mathrm{y}+\mathrm{A} \int_{0}^{2 \pi}((x(s)-\mathrm{Bx}(2 \pi-\mathrm{r})) \mathrm{ds}+\int_{0}^{2 \pi}\left(\mathrm{L}\left(x_{s}\right)+\mathrm{f}(\mathrm{s})\right) \mathrm{ds} \overbrace{=}^{*} \\
& \Rightarrow \mathrm{g}(0) \\
& \Rightarrow \mathrm{x}(2 \pi)=\mathrm{x}(0), \text { we conclude that } \mathrm{x}(.) \text { is a } 2 \pi-\mathrm{periodic} \text { weak }(\text { mild }) \mathrm{so}^{-}
\end{aligned}
$$
lution of Eq (1).

\section{Exemple :}

$$
\frac{d}{d t} \mathrm{x}(\mathrm{t})=\mathrm{A}(\mathrm{x}(\mathrm{t})-\mathrm{Bx}(\mathrm{t}-\mathrm{r}))+\mathrm{L} x_{t}+\mathrm{f}(\mathrm{t})
$$

let $\mathrm{A}$ be a closed linear operator and $\mathrm{X}$ be a UMD space, and

$$
\sup _{k}\left\|\left(i k I-A\left(I-B_{k}\right)\right)^{-1}\right\|=: \mathrm{M} \prec \infty \text { and }\|L\| \prec \frac{1}{r_{2 \pi}^{1 / p}} \text { then Eq (1) has }
$$
a unique weak solution.

we have ikI $-\mathrm{A}\left(\mathrm{I}-B_{k}\right)-L_{k}=\left[\mathrm{ikI}-\mathrm{A}\left(\mathrm{I}-B_{k}\right)\right]\left[\mathrm{I}-L_{k}\left(i k I-A\left(I-B_{k}\right)\right)^{-1}\right]$ it follows that ikI $-\mathrm{A}\left(\mathrm{I}-B_{k}\right)-L_{k}$ is invertible whenever $\left\|L_{k}\left(i k I-A\left(I-B_{k}\right)\right)^{-1}\right\| \prec 1[7$.Theorem 1.1.7] observe that $\left\|L_{k}\right\| \leq r_{2 \pi}^{1 / p}\|L\|$

Hence $\left\|L_{k}\left(i k I-A\left(I-B_{k}\right)\right)^{-1}\right\| \leq r_{2 \pi}^{1 / p}\|L\| \mathrm{M}:=\alpha$

Therefore, under the condition $\|L\| \prec \frac{1}{r_{2 \pi}^{1 / p} M}$

$$
\begin{aligned}
& \left(i k I-A\left(I-B_{k}\right)-L_{k}\right)^{-1}=\left[i k I-A\left(B_{k}\right)\right]^{-1}\left[I-L_{k}\left(i k I-A\left(I-B_{k}\right)^{-1}\right]^{-1}\right. \\
& =\left[i k I-A\left(B_{k}\right)\right]^{-1} \sum_{n=0}^{\infty}\left[L_{k}\left(i k I-A\left(I-B_{k}\right)^{-1}\right]^{n}\right.
\end{aligned}
$$

it follows that :

$\left\|i k\left(i k I-A\left(I-B_{k}\right)-L_{k}\right)^{-1}\right\| \leq\left\|i k\left(i k I-A\left(I-B_{k}\right)\right)^{-1}\right\| \sum_{n=0}^{\infty} \alpha^{n}$

$\leq \frac{M+1}{1-\alpha}$ then $\mathrm{ik} D_{k}$ is R-bounded. 


\section{Bibliographie}

[1] Hernan R.Henriquez,Michelle Pierri, Andrea ProkopczykPeriodic Solutions of abstract neutral functional differential equations, J. Math. Ana. Appl. 385 (2012) 608 - 621

[2] C.LizamaFourier multipliers and perodic solutions of delay equatons in Banach spaces,J . Math. Anal. Appl. 324 (2006) 921-933.

[3] W.Arend, S.Bu, The operator-valued Marcinkiewicz multiplier theorem and maximal regularity, Math.Z.240(2002),311-145.

[4] Y.Hino, T.Naito,N. Van Minh, J.S.Shin, Almost periodic solution of Differential Equations in Banach Spaces, Taylor and Francis, London,2002.

[5] J.Wu, Theory and Applications of Partial Differential Equations, Appl, Math .Sci. 119, Springer-verlag,19969.

[6] L. Weis : Operator-valued Fourier multiplier theorems and maximal Lpregularity. Preprint 2000.

[7] Khalil Ezzinbi; Lecture Notes on Differential Equations in Banach Spaces, African University of Science and Technology, 2009. 